\title{
The tuberculin skin test in relation to immunological in vitro reactions in BCG-vaccinated healthcare workers
}

\author{
H. Fjällbrant*, M. Ridell ${ }^{\#}$, L.O. Larsson*
}

The tuberculin skin test in relation to immunological in vitro reactions in BCGvaccinated healthcare workers. H. Fjällbrant, M. Ridell, L.O. Larsson. (C)ERS Journals Ltd 2001.

ABSTRACT: The aim was to study the tuberculin skin test in relation to immunological in vitro reactions in bacille Calmette-Guérin (BCG)-vaccinated healthcare workers.

The present study was performed in Sweden, a country with a low incidence of tuberculosis, a high BCG vaccination efficacy and high tuberculin conversion rates. BCG-vaccinated healthcare workers $(n=381)$ were tuberculin skin tested. From these, 11 subjects with negative tuberculin reactions $(<6 \mathrm{~mm})$ were matched for age and sex with 11 subjects with large positive reactions $(\geqslant 15 \mathrm{~mm})$. Lymphocyte transformation and the production of interferon-gamma (IFN- $\gamma$ ) were analysed after stimulation in vitro of peripheral blood mononuclear cells with tuberculin purified protein derivative, heatkilled tubercle bacilli and a culture filtrate from tubercle bacilli.

In the tuberculin-positive group the lymphocyte transformation response was 2-3 times larger, and IFN- $\gamma$ production was 7-10 times larger, than in the tuberculinnegative group $(\mathbf{p}<\mathbf{0 . 0 0 1})$.

The present results suggest that a positive tuberculin skin test in bacille CalmetteGuérin-vaccinated subjects indicates a stronger immune response of the protective T-helper 1-type than does a negative test. In similar settings, the study supports the traditional practice of regarding the tuberculin skin test in bacille CalmetteGuérin-vaccinated subjects as an indicator of a protective immune response against tuberculosis.

Eur Respir J 2001; 18: 376-380.
*Dept of Respiratory Medicine and Allergology, and ${ }^{\#}$ Dept of Microbiology and Immunology, Sahlgrenska University Hospital, Göteborg, Sweden.

Correspondence: H. Fjällbrant, Dept of Respiratory Medicine and Allergology, Sahlgrenska University Hospital, SE41345 Göteborg, Sweden.

Fax: 4631824904

Keywords: Bacille Calmette-Guérin vaccine

cellular immunity

occupational diseases tuberculin test

Received: October 52000

Accepted after revision April 42001

This study received generous financial support from the Oscar and Hanna Björkbom Fund and the Swedish Heart and Lung Foundation.
Pioneering work in Scandinavia showed that healthcare workers with a positive tuberculin skin test were more resistant to tuberculosis (TB) than those who were negative $[1,2]$. Other studies showed that the healthcare workers who were tuberculin positive after bacille Calmette-Guérin (BCG) vaccination were more resistant to TB than those who remained skin test negative $[3,4]$.

Subsequently, a positive tuberculin skin test has been used to indicate protection against $\mathrm{TB}$ in countries with BCG vaccination programmes [5-7]. This delayed-type hypersensitivity is, however, not identical to the protective immune response against TB [8], and its value as an indicator of a protective immune response is controversial [9-12].

An attractive method to evaluate the tuberculin skin test in this context is the use of surrogate markers for protective immunity. Interferon-gamma (IFN- $\gamma$ ) is a key cytokine for macrophage activation and enhanced intracellular killing of pathogens. Several findings point to the central role of a T-helper 1 (Th1) cell-derived IFN- $\gamma$ response in human TB defence [13-17]. Consequently, the amount of IFN- $\gamma$ production following lymphocyte stimulation with mycobacterial antigens has been suggested as a surrogate marker of vaccine-induced protective immunity [14, $18,19]$. The T-cell proliferation induced in this procedure, i.e. the lymphocyte transformation assay, correlates well with tuberculin skin test reactivity, but according to several studies it is a more sensitive indicator of mycobacterial sensitization [20, 21]. However, information on the relationship between the tuberculin skin test and surrogate markers for protective immunity in BCG-vaccinated subjects is scarce.

In order to assess the value of the tuberculin skin test as an indicator of a protective immune response against TB in BCG-vaccinated individuals, lymphocyte proliferation and IFN- $\gamma$ production induced by mycobacterial antigens in matched groups of tuberculinnegative and tuberculin-positive healthcare workers were analysed.

\section{Material and methods}

\section{Study population}

The tuberculin skin test was applied to 381 healthcare workers who had been BCG-vaccinated at birth. These included $325(85 \%)$ females. The mean age was 38.7 yrs. Eighty-five individuals $(22 \%)$ were tuberculin-negative, i.e. having induration of $<6 \mathrm{~mm}$ diameter, and 296 (78\%) were positive. One year from the initial test procedure, the tuberculin-negative 
subjects were retested before commencing the study. Eleven of those remaining negative $(0-5 \mathrm{~mm})$ were matched for age and sex with 11 subjects with large positive reactions $(15-20 \mathrm{~mm})$.

The mean tuberculin reactions of the two study groups were 2.2 and $15.8 \mathrm{~mm}$, respectively. The mean age of the 22 subjects was 38.0 yrs (range 27-46), and $18(82 \%)$ of those were female. Thus, the sex ratio and mean age of these 22 subjects was not significantly different from the original group of 381 . The subjects were healthy Swedes, with no coexisting medical conditions, and none were pregnant or breast feeding. All had been BCG-vaccinated at birth, and all had a BCG scar. Informed consent was obtained, and the study was approved by the local Ethics Committee.

\section{Skin testing}

An intradermal injection of 2 tuberculin units of tuberculin purified protein derivative (PPD) RT23 (Statens Seruminstitut, Copenhagen, Denmark) was given on the dorsal aspect of the right forearm. The reactions were read after 48-72 h. Induration of $<6 \mathrm{~mm}$ was defined as negative.

\section{Mycobacterial antigens}

In addition to tuberculin PPD RT23, antigens from two strains of Mycobacterium tuberculosis (4610/91 and 5109/95: Institute of Medical Microbiology, Göteborg University, Sweden) were used. These strains were isolated from Swedish patients in 1991 and 1995 , respectively, and showed all the characteristic properties of $M$. tuberculosis. They were grown on Sauton medium at $37^{\circ} \mathrm{C}$ for 6 weeks. A sterile culture filtrate was prepared from strain 5109/95, and a sterile suspension of wholes cells, homogenized by sonication, was prepared from strain 4610/91.

\section{Lymphocyte transformation assay}

The assay was performed simultaneously for the subjects in each matched pair, using a modification of a previously published technique [22]. For stimulation with mycobacterial antigens, $1 \times 10^{6}$ cells were added to the microtitre plates. For stimulation with the positive control concanavalin A $($ ConA $), 1 \times 10^{5}$ cells were used. Triplicates of each of the following stimulators were added: tuberculin PPD RT23, $10 \mu \mathrm{g} \cdot \mathrm{mL}^{-1}$; whole cells of $M$. tuberculosis, $10 \mu \mathrm{g} \cdot \mathrm{mL}^{-1}$; culture filtrate of $M$. tuberculosis, protein concentration $10 \mu \mathrm{g} \cdot \mathrm{mL}^{-1}$; Con A, $25 \mu \mathrm{g} \cdot \mathrm{mL}^{-1}$. A triplicate of wells with no stimulator was used as a negative control. The cells were then incubated in cell culture medium in $5 \%$ carbon dioxide $\left(\mathrm{CO}_{2}\right)$ at $37^{\circ} \mathrm{C}$ for 6 days. Culture supernatant $(150 \mu \mathrm{L})$ was removed from each well and frozen at $-70^{\circ} \mathrm{C}$ for later analysis of cytokine content. Fresh medium and stimulator $(150 \mu \mathrm{L})$ was added and the plate was incubated for another $24 \mathrm{~h}$. ${ }^{3} \mathrm{H}$-thymidine was then added and the plate was incubated overnight. The cells were harvested and radioactivity measured as counts per minute (cpm) using a $\beta$-counter (Matrix 96, Packard Instrument Co., Inc., Chicago, IL, USA). The proliferative response was calculated as the geometric mean cpm of the triplicates. The proliferative responses of stimulated cultures are presented as the difference $(\Delta \mathrm{cpm})$ between the radioisotope uptake in stimulated cultures and nonstimulated control cultures.

\section{Cytokine analysis}

IFN- $\gamma$ concentration was measured in supernatants from cells stimulated with the previously mentioned mycobacterial antigens and Con $\mathrm{A}$, as well as in supernatants from unstimulated cells, using a sandwich enzyme-linked immunosorbent assay (ELISA) technique as described by QUARFORDT et al. [22]. The maximum values given were $>10,000$ units $\cdot \mathrm{mL}^{-1}$. In calculations, these values were taken as being equal to 10,000 units $\cdot \mathrm{mL}^{-1}$.

\section{Statistical analysis}

The 22 subjects were matched in pairs, and due to the known large variation in the immunological assays, each pair was analysed simultaneously. The statistical calculations were based on the differences within the pairs. For comparisons between groups the Wilcoxon one-sample test was used. A p-value of $<0.05$ was considered as significant. Only subjects with small negative reactions and large positive reactions were selected to increase the possibility of detecting a significant difference between the two groups.

\section{Results}

\section{Lymphocyte transformation assay}

The proliferative responses of the negative controls, i.e. of unstimulated cultures, were $\leqslant 1,300 \mathrm{cpm}$ (median $500 \mathrm{cpm}$ ). The responses after stimulation with three mycobacterial antigens are shown in figure 1 . In the tuberculin-negative group, the median responses were $10-20$ times stronger than in unstimulated cultures $(9,100,9,300$ and $4,700 \mathrm{cpm}$ for tuberculin, heat-killed $M$. tuberculosis cells and $M$. tuberculosis culture filtrate, respectively). However, in the tuberculin-positive group the corresponding responses were 2-3 times stronger than in the tuberculin-negative group $(28,800,20,800$ and $12,700 \mathrm{cpm}, \mathrm{p}<0.001$ respectively). When the three antigens were compared, the lymphocyte transformation response to tuberculin PPD RT23 was similar to that with heat-killed $M$. tuberculosis, while the response to $M$. tuberculosis culture filtrate was significantly weaker.

\section{Cytokine production}

In unstimulated cultures, the IFN- $\gamma$ levels were close to zero. The IFN- $\gamma$ production after stimulation 


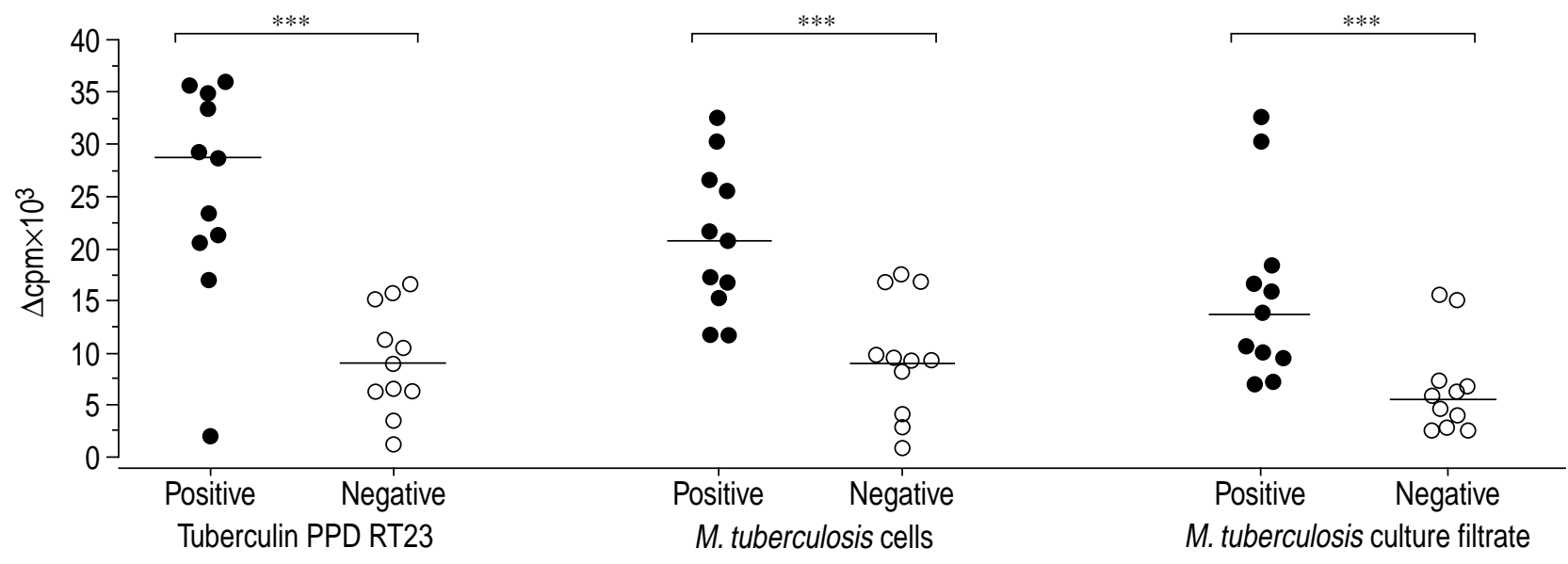

Fig. 1.-Proliferation of peripheral blood mononuclear cells from bacille Calmette-Guérin (BCG)-vaccinated healthcare workers with positive (O) and negative $(\bigcirc)$ tuberculin skin tests, after in vitro stimulation with tuberculin purified protein derivative (PPD) RT23, heat-killed Mycobacterium tuberculosis cells and a culture filtrate from $M$. tuberculosis. The individual values of proliferative responses are presented as the difference in counts per minute $(\Delta \mathrm{cpm})$ between the radioisotope uptake in stimulated cultures and nonstimulated control cultures. The median value in each group is indicated by a horizontal line. $* * *: \mathrm{p}<0.001$.

with the mycobacterial antigens is shown in figure 2 . In the tuberculin-negative group, the median IFN- $\gamma$ production was significantly higher than in unstimulated cultures $\left(700,700\right.$ and 600 units $\cdot \mathrm{mL}^{-1}$, respectively, for the three antigen stimulations). However, the corresponding IFN- $\gamma$ production in the tuberculinpositive group was $7-10$ times higher than in the tuberculin-negative group $(5,500,6,700$ and 4,400 units $\cdot \mathrm{mL}^{-1}, \mathrm{p}<0.001$ respectively). The differences between the three antigens in their ability to induce IFN- $\gamma$ production were not significant.

\section{Discussion}

In this study, the lymphocyte proliferation and IFN- $\gamma$ production induced by mycobacterial antigens was significantly stronger in a group of tuberculinpositive individuals than in a matched group of tuberculin-negative individuals, indicating a stronger immune response of the protective Th1-type in the tuberculin-positive group.

Tuberculin PPD RT23 was prepared in 1958 from several strains, including the $M$. tuberculosis type strain H37RV isolated in 1905. Considering the age of these antigens, preparations from two strains isolated from Swedish patients in the 1990s were also included in order to more accurately reflect the clinically relevant immunological responses of the subjects. However, the immunostimulatory effect of PPD RT23 was not less than that of the more recently isolated mycobacterial antigens.

A general BCG vaccination programme of newborns was in effect in Sweden until 1975. All subjects in the present study had been vaccinated at birth. Seven had been revaccinated, mostly $>20$ yrs ago. Many studies have shown considerable waning of tuberculin reactivity over time, after BCG vaccination

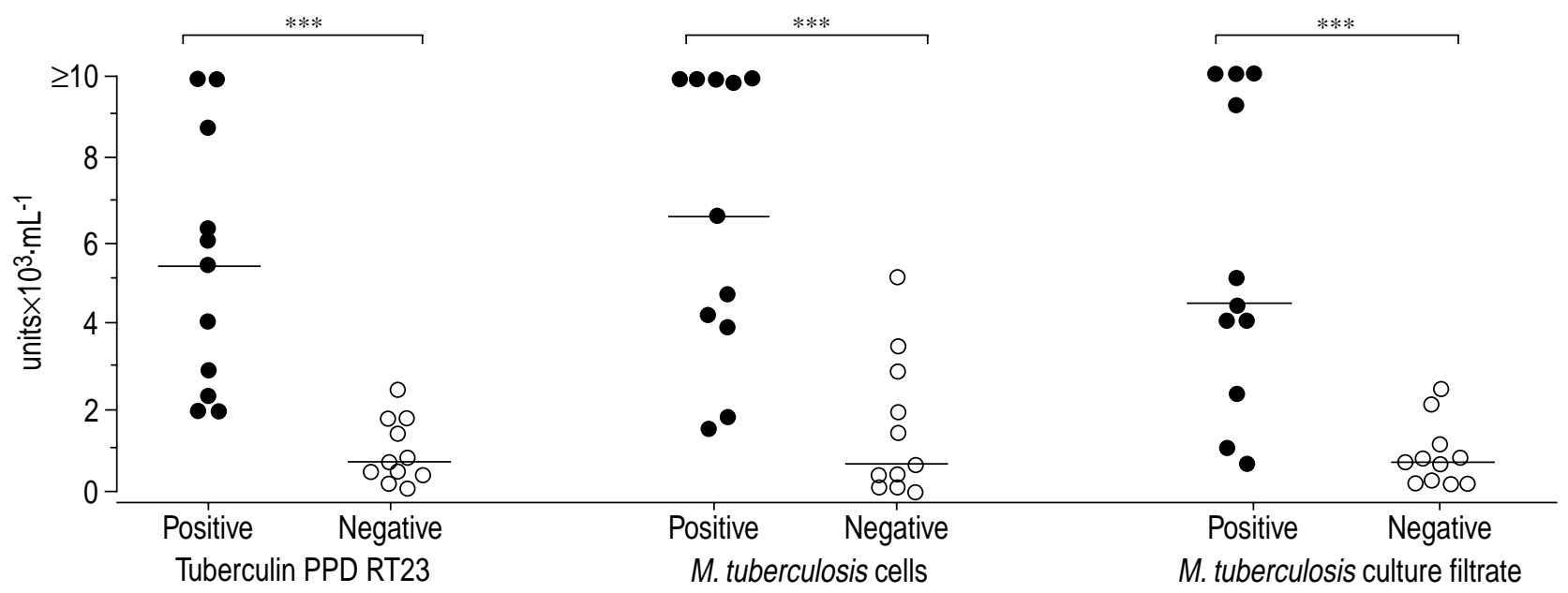

Fig. 2.-Concentrations of interferon-gamma (IFN- $\gamma)$ in supernatants from peripheral blood mononuclear cell cultures from bacille Calmette-Guérin (BCG)-vaccinated healthcare workers with positive $(\bullet)$ and negative $(\bigcirc)$ tuberculin skin tests, after in vitro stimulation with tuberculin purified protein derivative (PPD) RT23, heat-killed Mycobacterium tuberculosis cells and a culture filtrate from M. tuberculosis. The median value in each group is indicated by a horizontal line. $* * *: \mathrm{p}<0.001$. 
[23]. Nevertheless, and despite of the low incidence of TB in Sweden, $78 \%$ of the 381 healthcare workers who were initially tested had positive tuberculin reactions. Cross-reactions with the immune response to nontuberculous mycobacteria are likely to have contributed to these reactions [24], as well as to the in vitro results in the present study. Such reactions have been suggested to induce protection against TB as well $[25,26]$.

In vitro results supporting the concept of antigenspecific IFN- $\gamma$ production as an accurate indicator of vaccine responses have recently been published. Thus, BCG-induced lymphocyte transformation and IFN- $\gamma$ responses developed more rapidly in subjects previously sensitized to mycobacterial antigens than in nonsensitized subjects [14], which is to be expected by a memory $\mathrm{T}$-cell response. In addition, a study from South India found that despite tuberculin conversion after BCG vaccination, in vitro responses did not increase significantly, a finding consistent with the lack of BCG efficacy previously demonstrated in the same area [27].

A recently published review of mainly non-BCGvaccinated populations [28] showed the highest risk of TB in subjects with the largest tuberculin reactions. In several studies, however, the lowest risk was not registered among those with the smallest reactions, but in subjects with low-grade tuberculin reactions. In contrast, a review of controlled trials of BCG vaccination from different parts of the world showed no relationship between the average tuberculin conversion rate in each trial and the efficacy of the BCG strains [11]. However, a tendency for a positive correlation of tuberculin sensitivity and protection was found within each vaccine strain studied [11], e.g. in the British Medical Research Council trial [10] and a trial in western USA [29].

The presented results, indicating a relationship between vaccine-induced tuberculin reactivity and surrogate markers of protective immunity, are in accordance with those of a study from the USA performed 3 months after BCG vaccination [30], as well as 1-3 yrs after vaccination [19]. In contrast, the previously mentioned study from South India [27] showed no such relationship, pointing out the crucial importance of the demographic and environmental setting for the efficacy of BCG vaccination as well as for the significance of the tuberculin reaction.

Based on a large-scale BCG study in Malawi [12], the investigators drew the general conclusion that postvaccination tuberculin reactivity does not correlate with protection against TB. However, in countries such as Malawi, where BCG neither adds protection against TB nor increases tuberculin reactivity substantially, a relationship between postvaccination tuberculin reactions and BCG-derived protective immunity against $\mathrm{TB}$ is unlikely. In contrast, such a relationship is more likely to exist in settings like Scandinavia, in which both the efficacy of BCG and postvaccination tuberculin conversion rates [31] are high. It is suggested that a relationship between the postvaccination tuberculin reaction and protection against TB may exist in such settings, and that the results from countries such as Malawi cannot be used to draw general conclusions on this issue.

Protective immunity against tuberculosis is in all probability more complex than just the capacity to mount a T-helper 1 response. However, the presented results and studies reviewed in this paper support the traditional practice of regarding the tuberculin skin test as an indicator of protection against tuberculosis in bacille Calmette-Guérin-vaccinated subjects. To safeguard staff health, the exclusion of tuberculinnegative healthcare workers from the care of smearpositive patients seems appropriate, especially in cases of multidrug-resistant tuberculosis. These conclusions are relevant to settings such as those in the present study, but they may not be generally applicable.

\footnotetext{
Acknowledgements. The authors thank B. Andersson for valuable assistance concerning immunological assays, I-M. Doshé for her skillful and dedicated work, M. Magnusson and M. Jørgensen for help and discussions, and P. Nordin, for valuable statistical advice.
}

\section{References}

1. Birkhaug K. BCG vaccination in Scandinavia. Twenty years of uninterrupted vaccination against tuberculosis. Am Rev Tuberc 1947; 15: 234-249.

2. Rosenthal SR. Immunity in tuberculosis. In: Rosenthal SR. BCG vaccine: Tuberculosis-cancer. Littleton, Massachusetts, PSG Publishing Company, Inc, 1980; pp. 1-13.

3. Heimbeck J. Tuberculosis in hospital nurses. Tubercle 1936; 18: 97-99.

4. Scheel O. Tuberkulose blandt medisinske studenter og vaksinasjon med BCG. Nord Med 1935; 9: 841-846.

5. WHO Tuberculosis Research Office. Certain characteristics of BCG-induced tuberculin sensitivity. Bull World Health Organ 1955; 12: 123-141.

6. Turk JL. Relation between delayed hypersensitivity and cell-mediated immunity. J Royal Soc Med 1979; 72: 243-245.

7. Shaaban MA, Abdul Ati M, Bhahr GM, Stanford JL, Lockwood DNJ, McManus IC. Revaccination with BCG: its effects on skin tests in Kuwaiti senior school children. Eur Respir J 1990; 3: 187-191.

8. Dannenberg AM, Jr. Delayed-type hypersensitivity and cell-mediated immunity in the pathogenesis of tuberculosis. Immunol Today 1991; 12: 228-233.

9. Mackaness GB. Delayed hypersensitivity and its significance. In: International proceedings no. 14: States of immunization in tuberculosis in 1971. Washington D.C., DHEW, 1971; pp. 69-89.

10. Hart PD, Sutherland I, Thomas J. The immunity conferred by effective BCG and whole bacillus vaccines, in relation to individual variations in induced tuberculin sensitivity and to technical variations in the vaccines. Tubercle 1967; 48: 201-210.

11. Comstock GW. Identification of an effective vaccine against tuberculosis. Am Rev Respir Dis 1988; 138: 479-480.

12. Fine PEM, Sterne JAC, Pönnighaus JM, Rees RJW. Delayed-type hypersensitivity, mycobacterial vaccines and protective immunity. Lancet 1994; 344: 1245-1249. 
13. Mehra V, Gong JH, Iyer D, et al. Immune response to recombinant mycobacterial proteins in patients with tuberculosis infection and disease. J Infect Dis 1996; 174: 431-434.

14. Ravn P, Boesen H, Klarlund Pedersen B, Andersen P. Human $\mathrm{T}$ cell responses induced by vaccination with Mycobacterium bovis bacillus Calmette-Guérin. J Immunol 1997; 158: 1949-1955.

15. Ravn P, Pedersen BK. Mycobacterium avium and PPD-specific cytotoxicity mediated by CD4+ lymphocytes from healthy HIV seropositive and seronegative individuals. J Acquir Immune Defic Syndr Hum Retrovirol 1996; 12: 433-441.

16. Newport MJ, Huxley CM, Huston S, et al. A mutation in the interferon- $\gamma$-receptor gene and susceptibility to mycobacterial infection. $N$ Engl J Med 1996; 335: 1941-1949.

17. Condos R, Rom WN, Schluger NW. Treatment of multidrug-resistant pulmonary tuberculosis with interferon- $\gamma$ via aerosol. Lancet 1997; 349: 15131515.

18. Lein $\mathrm{AD}$, von Reyn CF. In vitro cellular and cytokine responses to mycobacterial antigens: application to diagnosis of tuberculosis and assessment of response to mycobacterial vaccines. $J$ Med Sci 1997; 313: 364 371.

19. Hoft DF, Tennant JM. Persistence and boosting of bacille Calmette-Guérin-induced delayed-type hypersensitivity. Ann Intern Med 1999; 131: 32-36.

20. Pabst HF, Godel JC, Spady DW, McKechnie J, Grace M. Prospective trial of timing of bacillus CalmetteGuérin vaccination in Canadian Cree infants. Am Rev Respir Dis 1989; 140: 1007-1011.

21. Smith JA, Reichman LB. Lymphocyte transformation. An aid in the diagnosis of tuberculosis in patients with nonreactive skin tests. Am Rev Respir Dis 1972; 106: 194-201.
22. Quarfordt I, Riise GC, Larsson S, et al. Immunological findings in blood and bronchoalveolar lavage fluid in chronic bronchitis patients with recurrent infectious exacerbations. Eur Respir J 1998; 11: 46-54.

23. Kröger, Katila ML, Korppi M, Brander E, Pietikäinen M. Rapid decrease in tuberculin skin test reactivity at preschool age after newborn vaccination. Acta Pediatr 1992; 1: 678-681.

24. Larsson LO, Bentzon MW, Lind A, et al. Sensitivity to sensitins and tuberculin in Swedish children. Part 5: a study of school children in an inland rural area. Tubercle 1993; 74: 371-376.

25. Fine PEM. Variation in protection by BCG: implications of and for heterologous immunity. Lancet 1995; 346: 1339-1345.

26. Hoft DF, Brown RM, Roodman ST. Bacille Calmette-Guérin vaccination enhances human $\gamma \delta$ $\mathrm{T}$ cell responsiveness to mycobacteria suggestive of a memory-like phenotype. J Immunol 1998; 161: 10451054.

27. Das SD, Narayanan PR, Kolappan C, Colston MJ. The cytokine response to bacille Calmette Guérin vaccination in South India. Int $J$ Tuberc Lung Dis 1998; 2: 836-843.

28. Watkins RE, Brennan R, Plant AJ. Tuberculin reactivity and the risk of tuberculosis: a review. Int J Tuberc Lung Dis 2000; 4: 895-903.

29. Aronson JD, Palmer CE. Experience with BCG vaccine in the control of tuberculosis among North American Indians. Public Health Rep 1946; 61: 802-820.

30. Kemp EB, Belshe RB, Hoft DF. Immune responses stimulated by percutaneous and intradermal bacille Calmette-Guérin. J Infect Dis 1996; 174: 113-119.

31. Beskow R, Bleiker M, Dahlström G, Mellbin T, Sjögren I, Styblo K. Tuberculin sensitivity in Swedish school children BCG vaccinated at birth. Lakartidningen 1981; 77: 118-120. 